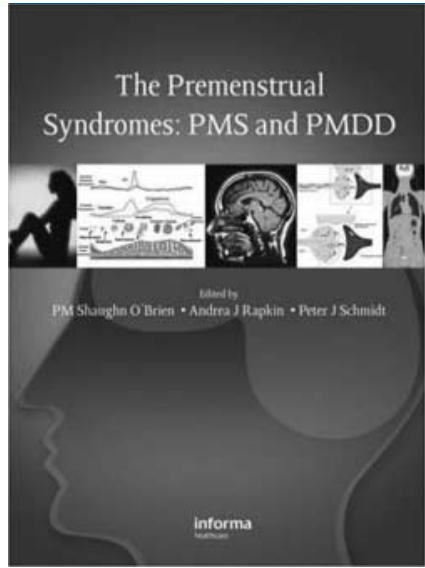

\section{The Premenstrual Syndromes: PMS and PMDD}

Edited by P. M. Shaughn O'Brien, Andrea Rapkin \& Peter J. Schmidt. Informa Healthcare. 2007. 192pp. f105.00 (hb). ISBN 9780415399746
Most books on premenstrual syndromes published in the past 5 years have been self-help books, a testament to the importance women place on their need to manage these symptoms. Anne Figart, a sociologist writing in 2005, noted that publicising and legitimising premenstrual syndrome (PMS) over the years has had a positive effect in increasing women's sense of control over their symptoms. However, she and others writing from a feminist perspective, also argue that the label PMS allows women to make excuses for their behaviour and permits doctors and scientists to exert control over them which might lead to bias and discrimination. In parallel with this debate, the scientific world has seen enormous advances in understanding the aetiology of PMS and premenstrual dysphoric disorder (PMDD), and the evidence base for treatment has grown.

A central theme of this book is the pulling together of a variety of perceptions of the nature of the disorders and their management from scientists, gynaecologists, psychiatrists and complementary medicine practitioners from the UK, Europe and the USA. The only missing voices in this text are those of women with PMS or PMDD whose attitudes to, and beliefs about, the available treatments are important.

Practice differs in several respects between disciplines and countries, sometimes following the evidence base but elsewhere departing from it. Even in the UK, some of drugs with the strongest evidence base are not licensed for treating PMS or PMDD, whereas some of those without demonstrated efficacy are licensed.

The book begins with a historical overview followed by a chapter examining the debate around these diagnoses and outlining the diagnostic criteria used in clinical settings and research. Elias Eriksson then reviews the role of serotonergic dysfunction in the pathophysiology of PMS. The other chapters on pathophysiology (ovarian steroids and endocrine studies, and $\gamma$-aminobutyric acid with reference to neuroimagaing studies) are found later in the book, with genetics the subject of the penultimate chapter. It would seem more logical to group the chapters relating to aetiology together and follow them with those relating to epidemiology, then assessment and treatment.

There are updates for psychiatrists who have forgotten the physiology of the menstrual cycle and gynaecologists who lack knowledge of neurotransmitters. Three chapters focus on epidemiology, comorbidity with other psychiatric conditions, and clinical presentation and course. Others discuss hormonal therapies, psychotropics (antidepressants with a paragraph on anxiolytics), ovarian cycle suppression and complementary therapies. Clinical assessment, evaluation and management are addressed. Nowhere else will you find such an up-to-date collection of scientific thought and evidence and I would recommend it as an essential reference for anyone working with women of reproductive potential.

Carol Henshaw Faculty of Health, Staffordshire University, Blackheath Lane, Stafford ST18 OAB, UK. Email: chenshaw@doctors.org.uk

doi: $10.1192 /$ bjp.bp.108.051870

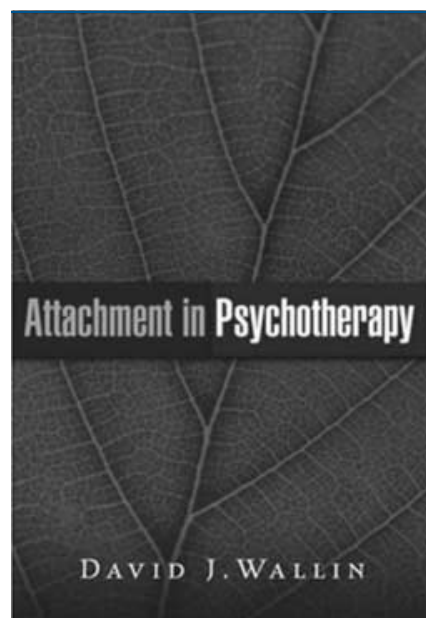

\section{Attachment in Psychotherapy}

By David I Wallin Guilford Press. 2007. 366pp. US\$39.00 (pb). ISBN 9781593854560

R. D. Laing opened one of his less enduring works with the warning: 'Few books today are forgivable'. Well, here's one to celebrate. If you practice psychotherapy and you read it, your patients stand to benefit. And, as an impressive synthesis of research and practice, it joins a handful of books that truly belong to this century rather than the previous one. Wallin has recognised that the techniques relationally minded therapists have been discovering may be out of step with some traditional clinical teaching, but can be usefully reconciled with findings from cognitive science and neuroscience as well as developmental psychology. Implications include the way therapeutic approaches are tailored throughout to an individual patient's attachment history and responses, the importance of non-verbal communications and how awareness of feelings in the body is fostered in therapy.

In the model Wallin develops, patients are helped to overcome the ways they have become, as a consequence of insecure early attachments, either embedded in or dissociated from their experience. They do this by developing the capacities to reflect, to mentalise and to be mindful. The therapist models and teaches these capacities within a therapeutic process whose aim is to bring about a lasting and liberating adjustment in patients' relationship to their experience. Minute attention is paid to the particulars of what is happening and how it is being understood, rather than relying on more abstract forms of interpretation. To work effectively, providing optimal conditions within a therapeutic relationship, therapists need the personal capacity to offer not only secure attachment but a very receptive and accepting attentiveness towards their patients. Well-chosen examples are provided of these in practice.

Like many good books, Attachment in Psychotherapy provides a snapshot of work in evolution. Its bland title belies its actual range. Its current organisation, which reveals progressive levels 
of subtlety of the author's model, rather than a sequence of therapeutic operations, may not be final. Perhaps two apparent omissions will be addressed in the next version. One is the role that eye movement desensitisation and reprocessing (EMDR) is finding, in other hands, in potentiating the sort of therapeutic operations that Wallin recommends. The other is the potential of therapeutic groups to afford an arena in which feelings not only emerge through what he terms evocations and enactments, but can also be understood and modulated within a rich but stable set of relationships. For now, we can be grateful that this book has arrived. It is much more than forgivable. It seems indispensable.

Chris Mace The Pines, St. Michael's Hospital., Warwick CV34 5QW, UK. Email: c.mace@warwick.ac.uk

doi: 10.1192/bjp.bp.107.04712 